\title{
Circ-SOX4 drives the tumorigenesis and development of lung adenocarcinoma via sponging miR-1270 and modulating PLAGL2 to activate WNT signaling pathway
}

Nan Gao ${ }^{1}$ and Baoguo Ye ${ }^{2^{*}}$

\begin{abstract}
Background: Lung adenocarcinoma (LUAD), a widespread histopathological subtype of lung cancer, is deemed as a malignant tumor with a peak risk of mortality. Emerged as RNA with a loop structure that depleted protein coding ability, circular RNA (circRNA) has been identified as a regulator in cancer progression. Circ-SOX4, identified as a novel circRNA, has not been studied in any cancer yet. Thus, the regulatory function that circ-SOX4 exerts on LUAD development remains obscure.
\end{abstract}

Aim of the study: This study aimed to investigate the biological function and molecular mechanism of circ-SOX4 in LUAD.

Methods: The expression of circ-SOX4 was detected by qRT-PCR. CCK-8, colony formation, transwell and wound healing assays were performed to explore the biological function of circ-SOX4 in LUAD. The interaction between miR1270 and circ-SOX41 (or PLAGL2) was confirmed by RNA pull down, luciferase reporter and RIP assays.

Results: Circ-SOX4 was found to be obviously upregulated in LUAD tissues and cells, and knockdown of it inhibited cell proliferation, invasion and migration in LUAD. Furthermore, silenced circ-SOX4 also inhibited LUAD tumor growth. Molecular mechanism assays revealed that circ-SOX4 interacted with miR-1270 in LUAD. Besides, PLAGL2 was confirmed as a downstream gene of miR-1270. Rescue assays validated that miR-1270 suppression or PLAGL2 overexpression countervailed circ-SOX4 depletion-mediated inhibition on cell proliferation, invasion and migration in LUAD. Additionally, it was discovered that circ-SOX4/miR-1270/PLAGL2 axis activated WNT signaling pathway in LUAD.

Conclusions: Circ-SOX4 boosted the development of LUAD and activate WNT signaling pathway through sponging miR-1270 and modulating PLAGL2, which provided a valuable theoretical basis for exploring underlying therapeutic target in LUAD.

Keywords: Circ-SOX4, miR-1270, PLAGL2, WNT, LUAD

*Correspondence: ye9369926692@163.com

2 Department of Anesthesiology, China-Japan Union Hospital of Jilin University, No. 126 XianTai Road, Changchun 130033, Jilin, China

Full list of author information is available at the end of the article

\begin{abstract}
Background
Lung cancer is a common type of malignancy and resulted in the death related to cancer worldwide [1]. The proportion of about $84 \%$ lung cancers is non-small cell lung cancer (NSCLC) [2]. However, lung adenocarcinoma (LUAD) is the most common kind of NSCLC with high morbidity and mortality [3]. To develop novel
\end{abstract}

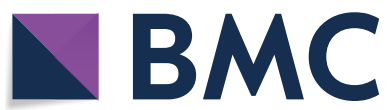

c) The Author(s) 2020. This article is licensed under a Creative Commons Attribution 4.0 International License, which permits use, sharing, adaptation, distribution and reproduction in any medium or format, as long as you give appropriate credit to the original author(s) and the source, provide a link to the Creative Commons licence, and indicate if changes were made. The images or other third party material in this article are included in the article's Creative Commons licence, unless indicated otherwise in a credit line to the material. If material is not included in the article's Creative Commons licence and your intended use is not permitted by statutory regulation or exceeds the permitted use, you will need to obtain permission directly from the copyright holder. To view a copy of this licence, visit http://creativeco mmons.org/licenses/by/4.0/. The Creative Commons Public Domain Dedication waiver (http://creativecommons.org/publicdomain/ zero/1.0/) applies to the data made available in this article, unless otherwise stated in a credit line to the data. 
treatments in LUAD, numerous efforts have been made over the past decades. However, the prognosis of LUAD patients remains unsatisfactory. As reported, the fiveyear-survival rate is under ten percent [4]. In consequence, identification of the effective diagnostic and therapeutic methods is essential for timely diagnosing and treating patients with $\operatorname{LUAD}[5,6]$.

Circular RNA (circRNA) is a particular type of noncoding RNA that contains multiple characteristics, including conservation, stabilization and tissue specific expression in living beings [7-9]. Numerous researches have confirmed the various regulatory mechanisms of circRNAs in cancer progression, like serving as sponges for miRNAs, forming RNA-protein complexes, and modulating the transcription of target genes $[10,11]$. Some circRNAs have been illustrated to play a key role in cancer progression. For instance, Hsa-circ_0068871 promotes cell proliferation and migration in bladder cancer by sponging miR-181a-5p [12]. Circ-SETD3 inhibits the growth of hepatocellular carcinoma via acting as a sponge of miRNA-421 [13]. Circ-LDLRAD3 functions as a diagnostic biomarker in pancreatic cancer [14]. There are a series of circRNAs were reported in LUAD. Hsa-circ_0001946 regulates miR-135a-5p/ SIRT1 axis in LUAD and enhances cell growth by activating Wnt pathway [15]. Hsa-circ_0006427 functions as a tumor suppressor in LUAD progression [16]. As a novel circRNA, circ-SOX4 has not been studied in LUAD. Therefore, the functional role and underlying mechanism of circ-SOX4 needs to be explored. The function of circ-SOX4 in LUAD was identified through both in vitro and in vivo experiments.

MicroRNAs (miRNAs), consisting 18-25 nucleotides, are a class of small RNAs with no coding ability, and exert essential function in the biological process [17]. As reported, miR-203a-3p facilitates cell proliferation and migration in colorectal cancer by targeting PDE4D [18]. microRNA-744 restrains the aggressive behaviors in glioblastoma by targeting NOB1 [19]. Former studies have illustrated that circRNAs affected tumor development by sponging specific miRNAs [20, 21]. For instance, hsa-circ-0005105 facilitates extracellular matrix degradation of chondrocyte via sponging miR26a [22]. Hsa-circ-0020397 regulates cell proliferation and metastasis in colorectal cancer by sponging miR138 expression [23]. MiR-1270 has been reported in thyroid cancer [24] and osteosarcoma [25] whereas it was not studied in LUAD.

Here, we analyzed the interaction between circ-SOX4 and miR-1270 by performing bioinformatics analysis and mechanism experiments. Consistently, the downstream mRNA and signaling pathway were explored. In summary, this study unveiled that circ-SOX4 promotes
LUAD development via targeting miR-1270/PLAGL2 axis and activating WNT pathway, which might be helpful for exploring the new strategies to treat patients with LUAD.

\section{Materials and methods Clinical tissue specimens}

Total LUAD tissues and adjacent normal tissues were obtained from China-Japan Union Hospital of Jilin University from May 2016 to August 2018. Before surgical resection, all involved participants signed informed consent. Afterwards, samples were frozen and stored at $-80{ }^{\circ} \mathrm{C}$ for further use. The procedures of this study were authorized by China-Japan Union Hospital of Jilin University.

\section{Microarray analyses}

To begin with, RNase R (Epicentre, Inc., WI, USA) was applied to treat with total RNAs for removing linear RNAs and enriching circRNAs. Then, we amplified the enriched circRNAs and thereby transcribed these circRNAs into fluorescent cRNA using a random priming method (Arraystar, MD, USA). Afterwards, the labeled cRNAs were mixed into the Arraystar Human circRNA Array V2 $(8 \times 15 \mathrm{~K}$, Arraystar $)$. After the slides being rinsed, the Agilent G2565CA Microarray Scanner System (Agilent Technologies, CA, USA) was utilized to scan the arrays. Agilent Feature Extraction software was employed to process the acquired array images. Quantile normalization was conducted by Arraystar software. Fold-change filtering identified differently expressed circRNAs between two groups.

\section{Cell culture and transfection}

LUAD cell lines (A549,SPC-A1,H1299, PC-9; ATCC) and one normal human lung epithelial cell line (BEAS2B; ATCC) were cultured in RPMI1640 medium (Gibco), supplemented with $10 \%$ fetal bovine serum (FBS; Gibco/ Invitrogen Inc., USA) at $37{ }^{\circ} \mathrm{C}$ with $5 \%$ humidified $\mathrm{CO}_{2}$. For RNase R treatment, RNA was cultured at $37{ }^{\circ} \mathrm{C}$ for 15 min with or without RNase R (Epicentre Technologies, USA).

GenePharma (Shanghai, China) offered shRNAs targeting circ-SOX4 (sh-circ-SOX4\#1/2) and negative control (sh-NC), pcDNA3.1 as well as pcDNA3.1/PLAGL2. MiR1270 inhibitor, inhibitor control (NC inhibitor), miR1270 mimics and mimics control (NC mimics) were also acquired from GenePharma. Lipofectamine 2000 Reagent (Invitrogen) was applied to conduct cell transfection in line with the protocols of the manufacturer. 


\section{qRT-PCR}

Under the suggestions of manufacturer, total RNA extracted from LUAD cells was isolated with TRIzol reagent (Invitrogen). The isolated RNAs $(1 \mu \mathrm{g})$ were reverse-transcribed into cDNA by the use of TaqManTM Advanced miRNA cDNA Synthesis Kit (Waltham, MA, USA) or the reverse transcription kit (Takara, Otsu, Japan). qRT-PCR was carried out by employing the StepOneTM Real-Time PCR System and the SYBR ${ }^{\circledR}$ Green Mixture (Takara). GAPDH or U6 was acted as the internal control. The $2^{-\Delta \Delta C t}$ method was utilized to quantify relative expression levels.

\section{CCK-8 assay}

The proliferation of LUAD cell was detected through a Cell Counting Kit-8 (Dojindo Molecular Technologies, Japan). $1 \times 10^{3}$ cells were added into a 96-well plate, followed by the incubation of 24, 48, 72 and 96 h. Afterwards, each well was added with $10 \mu \mathrm{l}$ CCK- 8 solution, followed by another $4 \mathrm{~h}$ of incubation. The absorbance was detected at $450 \mathrm{~nm}$ using a spectrophotometer (Olympus, Japan).

\section{Colony formation assay}

LUAD cells were placed in 6-well plates for an incubation of 2 weeks. Then, the methanol was utilized to fix the colonies for $30 \mathrm{~min}$, and $0.1 \%$ crystal violet was applied to stain the colonies for $20 \mathrm{~min}$ as well as PBS was employed to wash the colonies three times. The number of colonies was manually counted.

\section{Wound healing assay}

Wound-healing assay was carried out to measure cell migration. To begin with, cells were trypsinized and added into 6-well plates. After $12 \mathrm{~h}$, pipette tip was employed to make an artificial wound. $0 \mathrm{~h}$ and $24 \mathrm{~h}$ after wounding, the wound width was examined, respectively.

\section{Transwell assay}

A 24-well transwell chamber $(8 \mathrm{~mm}$, Corning Life Sciences, Corning, USA) was utilized in transwell assay. Matrigel (BD Biosciences, USA) coated the upper surface of the membrane. The top chamber was introduced with a total of $1 \times 10^{3}$ cells suspended in serum-free medium, and $10 \%$ FBS was added into the lower chamber. Being cultured for $24 \mathrm{~h}$, cells on the upper membrane surface were carefully removed. Afterwards, methanol was utilized to fix the lower membrane surface and $0.1 \%$ crystal violet was applied to stain the surface. Finally, a microscope was applied to calculate the number of invaded cells.

\section{Subcellular fractionation assay}

PARIS $^{\mathrm{TM}}$ Kit (Ambion, Austin, TX, USA) was applied to isolate nuclear fraction from cytoplasm fraction under the instructions of the manufacturer. A549 and SPCA1 cell lines were eluted twice, dissolved in cell fraction buffer. Afterwards, the supernatant was collected after centrifugation. Subsequently, PBS was utilized to rinse the left lysate for five times with and then the lysate was centrifuged. qRT-PCR analyzed the extracted RNAs, normalizing to U6 (nucleus control) and GAPDH (cytoplasm control).

\section{RNA immunoprecipitation (RIP) assay}

To begin with, A549 and SPC-A1 cells were dissolved in RIP lysis buffer. Subsequently, they were incubated with RIP buffer that contains magnetic beads coated with $\mathrm{Ago}_{2}$ antibodies (Abcam, Cambridge, MA, USA) or IgG antibodies at $4{ }^{\circ} \mathrm{C}$ overnight. RNA was then purified and quantified by qRT-PCR.

\section{Luciferase reporter assays}

The circ-SOX4-WT, circ-SOX4-Mut-1 and circ-SOX4Mut-2, PLAGL2-WT, PLAGL2-Mut reporters were acquired from GeneArt ${ }^{\mathrm{TM}}$ Site-Directed Mutagenesis System (Thermo Fisher Scientific). Lipofectamine 2000 was utilized to co-transfect miR-1270 mimics or NC mimics with the constructed luciferase reporters in A549 or SPC-A1 cells. Luciferase activities were evaluated with a Dual Luciferase Assay Kit (OMEGA Engineering Inc.) after $48 \mathrm{~h}$ of incubation.

Biovector NTCC Ltd (Beijing, China) offered the TOP and FOP flash luciferase reporter vectors. Sh-NC, sh-circ-SOX4 or different concentration of pcDNA3.1/ circ-SOX4 or was co-transfected with TOP Flash and FOP flash reporters into A549 or SPC-A1 cells with the employment of Lipofectamine 2000. After 48 h, Dual Luciferase Assay Kit was applied to evaluate the activity of Wnt/ $\beta$-catenin signaling pathway.

\section{RNA pull-down assay}

Circ-SOX4 RNAs were transcribed in vitro and biotinlabeled with Biotin RNA Labeling Mix (Roche). Biotinylated RNAs were cultured with protein extracted from A549 or SPC-A1 cells. Magnetic beads were utilized to mix with each binding reaction, and then the beads were rinsed with washing buffer. SDS-PAGE was employed to separate the circRNA-interacting proteins. After the gels being silver stained, the proteins were subjected to Western blot analysis.

\section{Western blot}

Proteins were obtained from cell lysis, and then separated by SDS-PAGE. Later, they were transferred onto 
PVDF membranes. 5\% defatted milk was applied to seal the immunoblots for $1 \mathrm{~h}$. Next, primary antibodies were used to incubate with the membranes at $4{ }^{\circ} \mathrm{C}$ overnight and then the membranes were cultured with secondary antibodies at room temperature for another $1 \mathrm{~h}$. Finally, the immunoblots were detected with BioImaging Systems (BIO-RAD, CA, USA).

\section{Xenograft tumors in nude mice}

Male nude BALB/c mice aged 4-5 weeks and weighted $20 \mathrm{~g}$ were provided from Vital River Laboratory Animal Technology (Beijing, China). SPC-A1 cells transfected with sh-circ-SOX4 or sh-NC were subcutaneously injected into nude mice. The volume of tumor xenografts that formed in nude mice was detected every 4 days. Four weeks later, all the animal experimental protocols were approved by China-Japan Union Hospital of Jilin University.

\section{Immunohistochemical analysis}

The paraffin-embedded tissues were deparaffinized, rehydrated and treated by $0.3 \% \mathrm{H}_{2} \mathrm{O}_{2}$ for antigen retrieval. For staining, the tissues were treated with $\mathrm{Ki}-67$ antibodies (Abcam, Cambridge MA). Ruptured by nuclear membrane for $30 \mathrm{~min}$ with $0.1 \%$ Triton X-100 and blocked with $5 \%$ normal donkey serum, slides were separately incubated with primary antibody and secondary antibody. The results were visualized with Olympus BX 41 Microscope (Olympus Corporation, Japan).

\section{Statistical analysis}

SPSS 21.0 software (IBM, Armonk, USA) was utilized for data analyses. Student's t-test was applied for evaluating differences of two groups and one-way ANOVA for more than two groups. Data were confirmed as mean \pm standard deviation (SD). $\mathrm{P}<0.05$ was deemed as statistically significant. Each experiment was repeated three times.

\section{Results}

Circ-SOX4 is highly expressed and knockdown of it hampers cell growth in LUAD

To investigate the potential functional circRNAs in LUAD, microarray analysis was utilized to assess the expression of circRNAs in LUAD tissues and adjacent non-tumor tissues (Fig. 1a). Then circ-SOX4, which was mostly significantly upregulated in LUAD tissues, was chosen to conduct qRT-PCR assay. And one normal lung epithelial cell line (BEAS-2B) acted as a control. The result displayed that circ-SOX4 expression was dramatically upregulated in LUAD cell lines (A549, SPC-A1, H1299 and PC-9) (Fig. 1b). Additionally, schematic diagram displayed the genomic location and splicing pattern of circ-SOX4 (Fig. 1c). Moreover, the fragment of linear
SOX4 mRNA was digested by RNase R, whereas circSOX4 remained after the treatment of RNase R (Fig. 1d). Subsequently, several experiments were applied to probe the biological function of circ-SOX4 in LUAD. With the employment of qRT-PCR analysis, a notably lower expression of circ-SOX4 was observed in LUAD cells with the transfection of sh-circ-SOX4 than that in sh-NCtransfected cells (Fig. 1e). Importantly, we found that the mRNA expression of SOX4 showed no evident changes in cells transfected with sh-circ-SOX4 (Additional file 1: Figure S1A). Later, CCK-8 and colony formation assays showed that circ-SOX4 deficiency repressed the proliferation ability of A549 and SPC-A1 cells (Fig. 1f-g). Afterwards, knockdown of circ-SOX4 weakened the invasion capability of A549 and SPC-A1 cells (Fig. 1h). Besides, the migration of A549 and SPC-A1 cells was repressed by circ-SOX4 knockdown (Fig. 1i). Further, we performed in vivo experiments to assess the effect of circ-SOX4 on the tumor growth of LUAD. As demonstrated in Fig. 2a, tumors derived from circ-SOX4 silenced LUAD cell were remarkably smaller than those in NC group. Consistently, the tumor volume and weight were also smaller in shcirc-SOX4 group compared with sh-NC group (Fig. 2b, c). Results of IHC assay revealed that ki-67 expression was downregulated after circ-SOX4 was stably silenced (Fig. 2d). To sum up, circ-SOX4 is highly expressed and circ-SOX4 depletion represses cell proliferation, invasion, migration and tumor growth in LUAD.

\section{Circ-SOX4 sponges miR-1270 in LUAD}

With the intention of further probing the molecular mechanism of circ-SOX4 in LUAD, subcellular fractionation assay was conducted to determine the distribution of circ-SOX4 in cytoplasm and nucleus, and the result suggested that circ-SOX4 mainly localized in cytoplasm (Fig. 3a). In addition, circ-SOX4 was found to enrich in $\mathrm{AgO}_{2}$ antibody group but not in IgG antibody group (Fig. 3b). Existing investigations have confirmed that circRNAs contribute to cancer progression by sponging specific miRNA [26, 27]. Therefore, circular RNA interactome database was searched to predict potential miRNAs for circ-SOX4, and miR-1282, miR-1304, miR-1307, miR-432, miR-488, miR-1270, miR-620, miR-626, miR636 and miR-646 were found. RIP analysis delineated that miR-1270 was the most enriched in $\mathrm{Ago}_{2}$ antibody group compared with other miRNAs (Fig. 3c). Herein, miR-1270 was chosen to do the following experiments. qRT-PCR assay indicated that circ-SOX4 depletion significantly increased miR-1270 expression in LUAD cells (Fig. 3d). In addition, we tested the transfection efficiency of miR-1270 mimics in LUADA cells. As a result, miR1270 mimics remarkably increased miR-1270 expression (Fig. 3e). Furthermore, the luciferase activity of vector 


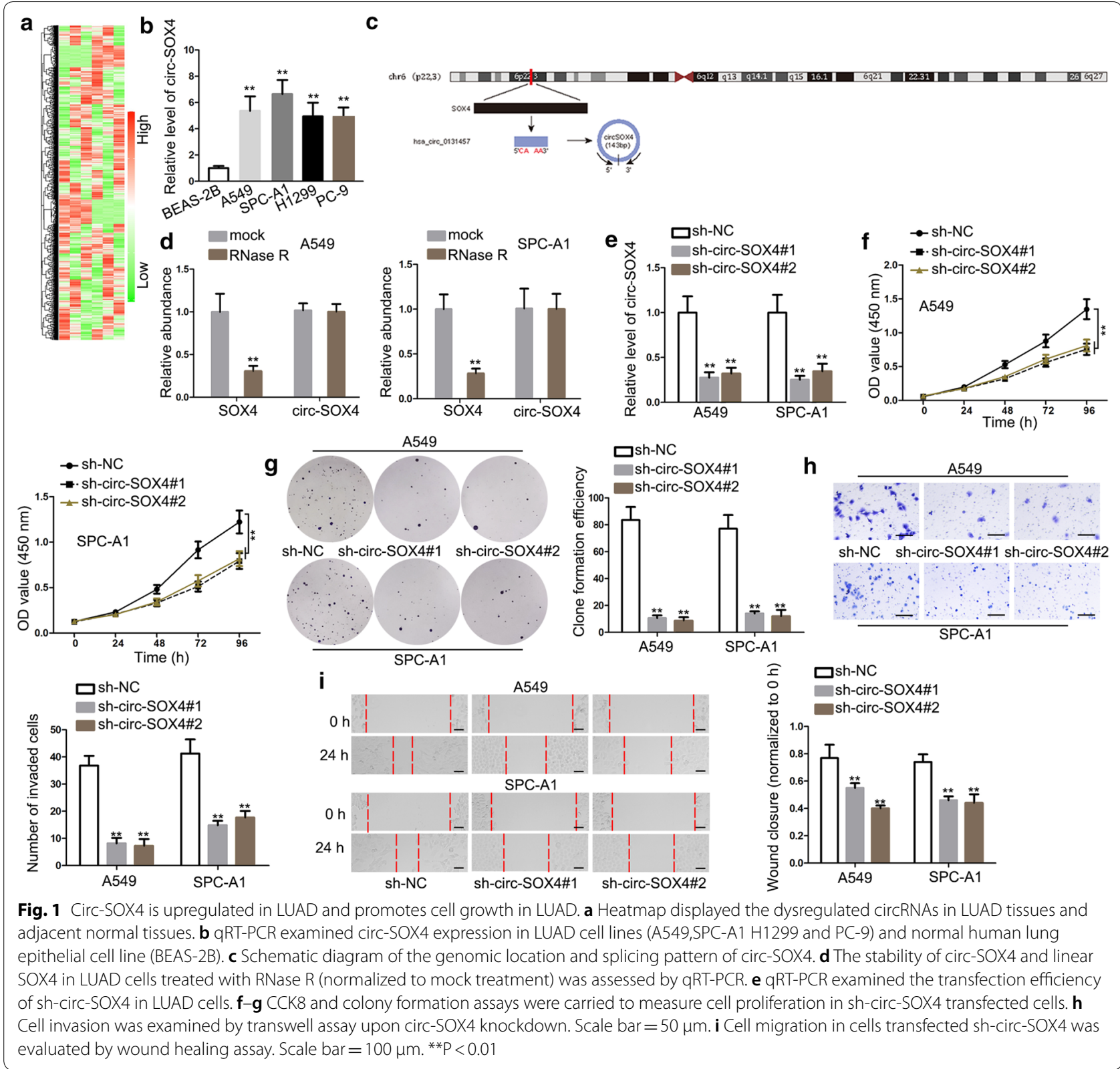

containing circ-SOX4 sequence observably decreased by miR-1270 mimics, suggesting the potential interaction between circ-SOX4 and miR-1270 (Fig. 2f). Through searching starBase, it was predicted that miR-1270 had two binding sites for circ-SOX4 (Fig. 3g). Luciferase reporter assay further confirmed that the circ-SOX4 interacted with miR-1270 in the above two binding sites (Fig. 3h). What's more, RNA pull down assay validated that circ-SOX4 bind to miR-1270 (Fig. 3i). Overall, circSOX4 functions as a sponge for miR-1270 in LUAD.
PLAGL2 is a target gene of miR-1270 in LUAD

Afterwards, through searching PITA, RNA22, miRmap and microT databases, the potential target gene of miR1270 was displayed by Venn diagram and the predicted mRNAs were PLAGL2, TCF12 and GLI3 (Fig. 4a, b). qRT-PCR analysis showed that PLAGL2 expression was evidently upregulated in LUAD cell lines whereas the expression of TCF12 and GLI3 showed no remarkable difference (Fig. 4c). qRT-PCR analysis and western blot assay depicted that PLAGL2 mRNA and protein expressions were significantly reduced by miR- 1270 overexpression or circ-SOX4 silencing (Fig. 4d, e). As displayed in 

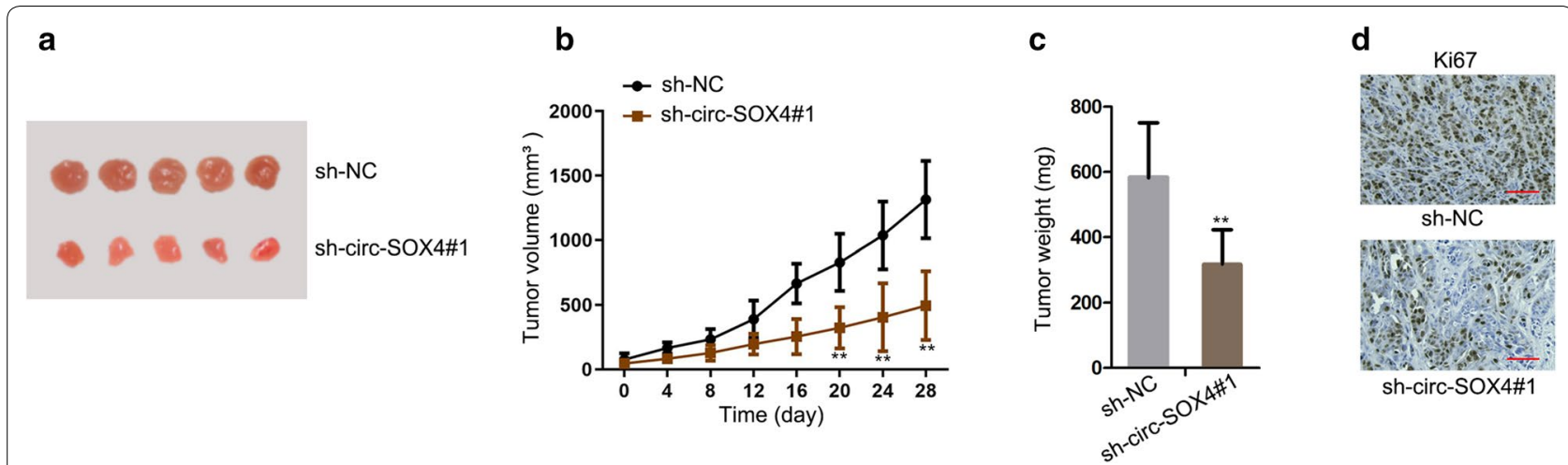

Fig. 2 Circ-SOX4 promotes LUAD cell growth in vivo. a Tumors removed from mice in two groups were presented. $\mathbf{b}$, c The tumor volume and weight in the groups of sh-NC and sh-circ-SOX4 were showed. $\mathbf{d}$ The expression of ki-67 was detected by $\mathrm{IHC}$ assay. ${ }^{* *} \mathrm{P}<0.01$

Fig. 4f, miR-1270 had a binding site for PLAGL2. Then, luciferase reporter assay demonstrated that the luciferase activity of PLAGL2-WT was observably decreased by miR-1270 mimics while that of PLAGL2-Mut depicted no notable change (Fig. 4g). Finally, circ-SOX4, miR-1270 and PLAGL2 were all aggregated in $\mathrm{Ago}_{2}$-conjugated beads via RNA pull down assay (Fig. 4h). Taken together, PLAGL2 is a target gene of miR-1270 in LUAD.

\section{Circ-SOX4 promotes LUAD cell growth by sponging miR-1270 and upregulating PLAGL2 expression}

To testify whether circ-SOX4 promotes LUAD development by sponging miR-1270 and upregulating PLAGL2 expression, rescue assays were carried out. Before conducting rescue experiments, the transfection efficiency of miR-1270 inhibitor and pcDNA-PLAGL2 were measured by qRT-PCR in APC-A1 cells. The results depicted that miR-1270 inhibitor evidently inhibited miR-1270 expression and PLAGL2 expression was conspicuously enhanced by transfecting pcDNA-PLAGL2 (Fig. 5a). Then, CCK-8 and colony formation assays demonstrated that miR-1270 suppression or PLAGL2 overexpression countervailed circ-SOX4 depletion-mediated inhibitive effect on cell proliferation (Fig. 5b, c). Besides, inhibitory function caused by circ-SOX4 deficiency on cell invasion was recovered by miR-1270 suppression or overexpressed PLAGL2 (Fig. 5d). What's more, it was confirmed by wound healing assay that the inhibition of miR-1270 or PLAGL2 upregulation offset the inhibitive effect of circ-SOX4 knockdown on cell migratory ability (Fig. 5e). In summary, circ-SOX4 promotes LUAD cell proliferation, invasion and migration by sponging miR-1270 and upregulating PLAGL2 expression.

\section{Circ-SOX4/miR-1270/PLAGL2 signal axis activates WNT pathway in LUAD}

According to existing literature, PLAGL2 has been reported to activate WNT pathway in colorectal adenocarcinoma [28]. To find out whether circ-SOX $4 / \mathrm{miR}$ 1270/PLAGL2 signal axis activated WNT pathway in LUAD, the following experiments were performed. Through TOP/FOP flash assay, we observed that the activity of WNT pathway was effectively promoted with the increasing concentration of pcDNA-circ-SOX4 (Fig. 6a). Further, the activity of WNT pathway was restrained by circ-SOX4 deficiency (Fig. 6b). Lastly, qRTPCR and western blot assays displayed that repressed miR-1270 or upregulated PLAGL2 reserved circ-SOX4 depletion-mediated effect on the mRNA and protein expressions of CTNNB1 ( $\beta$-catenin), CCND1, CDK2, c-MYC and MMP2, which were known as the relative genes of WNT pathway (Fig. 6c, d). Further, we detect the protein level of CD44, E-cadherin, and N-cadherin for checking epithelial-mesenchymal transition (EMT). The results displayed that the inhibition of miR-1270 or overexpressed PLAGL2 increased the protein level of CD44 and $\mathrm{N}$-cadherin whereas reduced that of $\mathrm{E}$-cadherin in sh-circ-SOX4 transfected cells (Additional file 1: Figure S1B). According to previous reports, we found that CD44 and EMT were closely correlated with WNT pathway [29-32]. All the findings suggest that circ-SOX4/miR1270/PLAGL2 signal axis activates WNT pathway in LUAD.

\section{Discussion}

Despite various advances in the treatment of LUAD, it is still one of the main reasons of death globally [33]. For the past few years, the cases of LUAD increased annually and ascended to the highest morbidity kind of NSCLC [34]. A growing number of studies have indicated that the aberrant expression of circRNAs displayed regulatory 


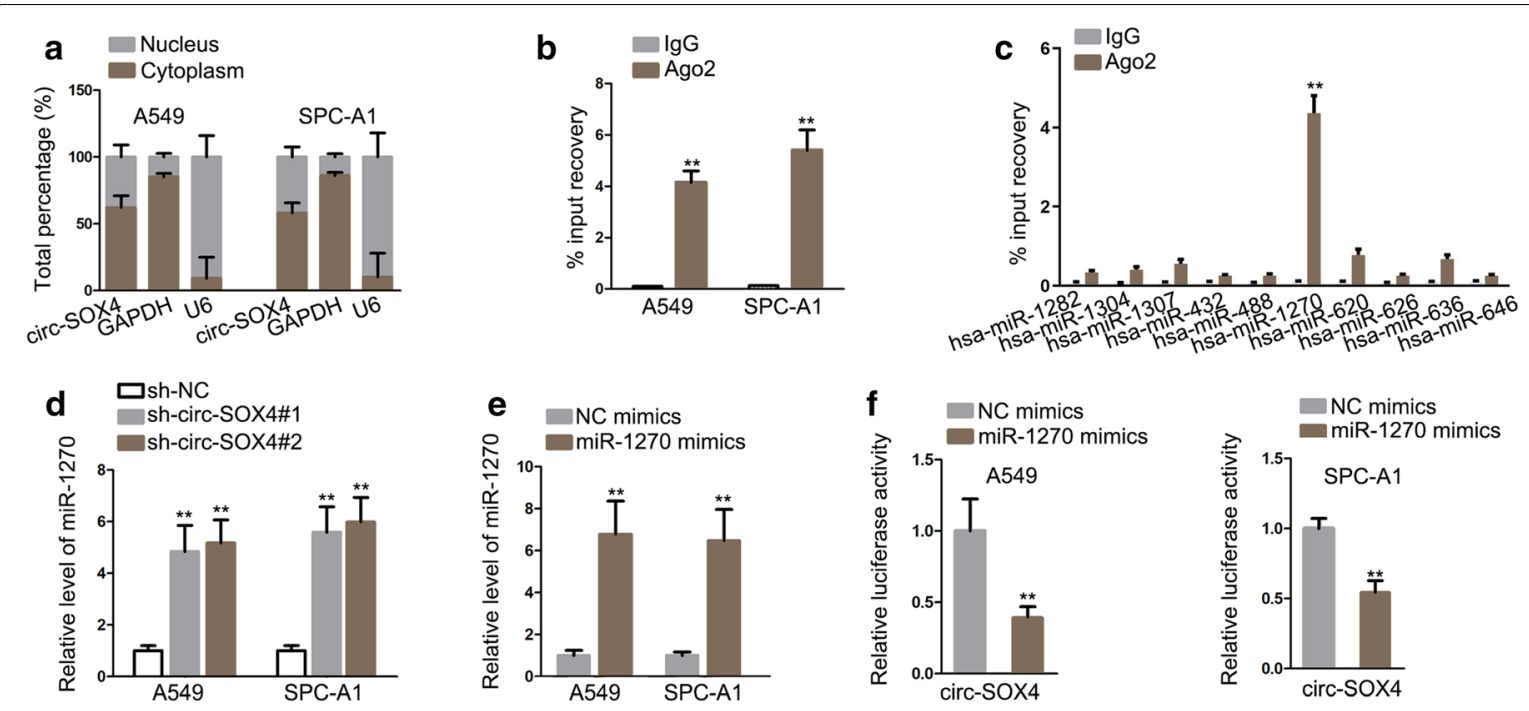

g

circ-SOX4 WT-1: 5' A U G C A C A A C G C C G A G A U C U C C A A 3' circ-SOX4 WT-2: 5' G C C C G A C A U G C A C A A A C G C C G A G A 3'

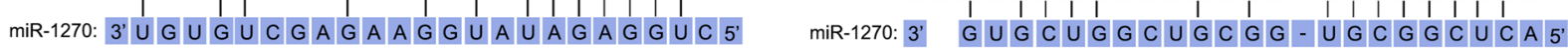
circ-Sox4 Mut1: $5^{\prime} \cup \cup \cup$ G G U C A A G G C G G U G U A G A G G U A 3' circ-sox4 Mut2: 5' G G C G C U G A U G G A G A U G C G G C U C A 3'
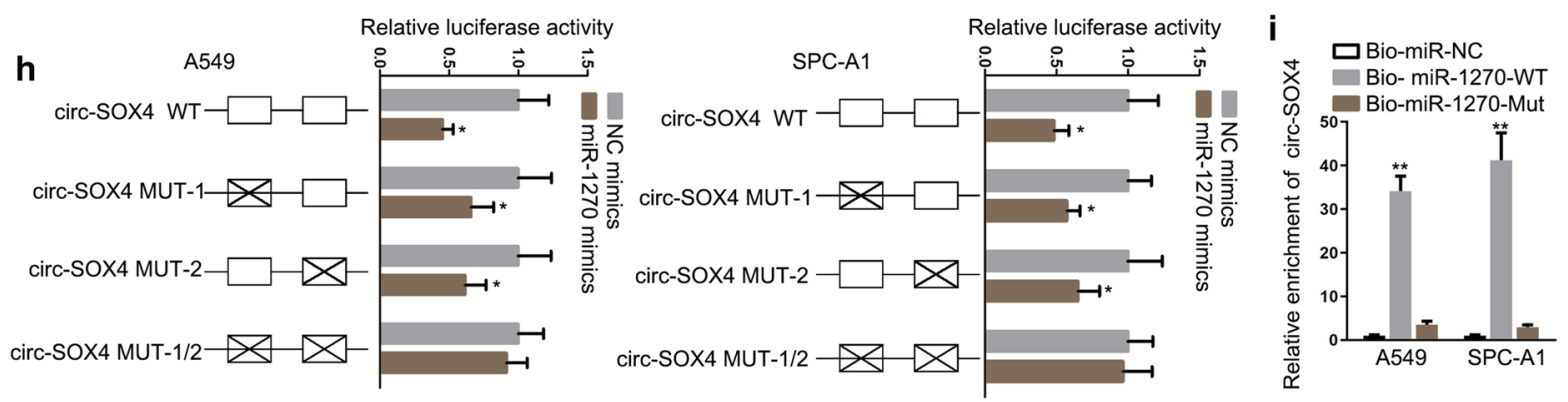

Fig. 3 Circ-SOX4 sponges miR-1270. a Subcellular fraction assay was carried out to examine the distribution of circ-SOX4 in nuclear and cytoplasm of A549 and SPC-A1 cells. b RIP assay was performed to confirm that circ-SOX4 could bind with RNA-induced silencing complex (RISC). c Several miRNAs that possess the potential of binding with circ-SOX4 were predicted by circular RNA interactome database and screened out by RIP assay d The influence of circ-SOX4 deficiency on miR-1270 expression was evaluated by qRT-PCR. e The overexpression efficiency of miR-1270 was examined in LUAD cells. $\mathbf{f}$ The effect of miR-1270 overexpression on the luciferase activity of reporter containing circ-SOX4 sequence was assessed by luciferase reporter assay. $\mathbf{g}$ The predicted binding sites between miR-1270 and circ-SOX4 were depicted. $\mathbf{h}-\mathbf{i}$ Luciferase reporter and RNA pull down assays were conducted to testify the binding ability between miR-1270 and circ-SOX4. ${ }^{*} \mathrm{P}<0.05$, ${ }^{* *} \mathrm{P}<0.01$, ${ }^{* * *} \mathrm{P}<0.001$

roles in diverse cancers [35-38]. In the current investigation, we chose an upregulated circRNAs in LUAD tissues through a microarray analysis. To our knowledge, circ-SOX4 was firstly investigated in LUAD. Moreover, we observed high expression of circ-SOX4 in LUAD tissues and cell lines. Functionally, high level of circRNAs might exert regulatory functions in LUAD. As recently reported, dysregulation of circRNAs can contribute to the malignant behaviors of human cancer cells. For instance, high level of hsa_circ_0000515 acts as a tumor promoter in cervical cancer through miR-326/ELK1 axis [39]. CircRNA-ENO1 and its linear mRNA ENO1 promotes glycolysis and tumor formation in LUAD [40]. Circular form of ERBB2 promotes the malignant process in gallbladder cancer [41]. In this study, we also designed and performed functional experiments to determine the role of circ-SOX4 in LUAD cellular processes. After silencing of circ-SOX4, we determined that circ-SOX4 knockdown hampered cell proliferation, invasion and migration in LUAD. In vivo experiments showed that silenced circ-SOX4 was correlated with the decreased tumor size, volume, weight, and ki-67 expression. In consequence, it was confirmed that circ-SOX4 accelerated the development of LUAD. 


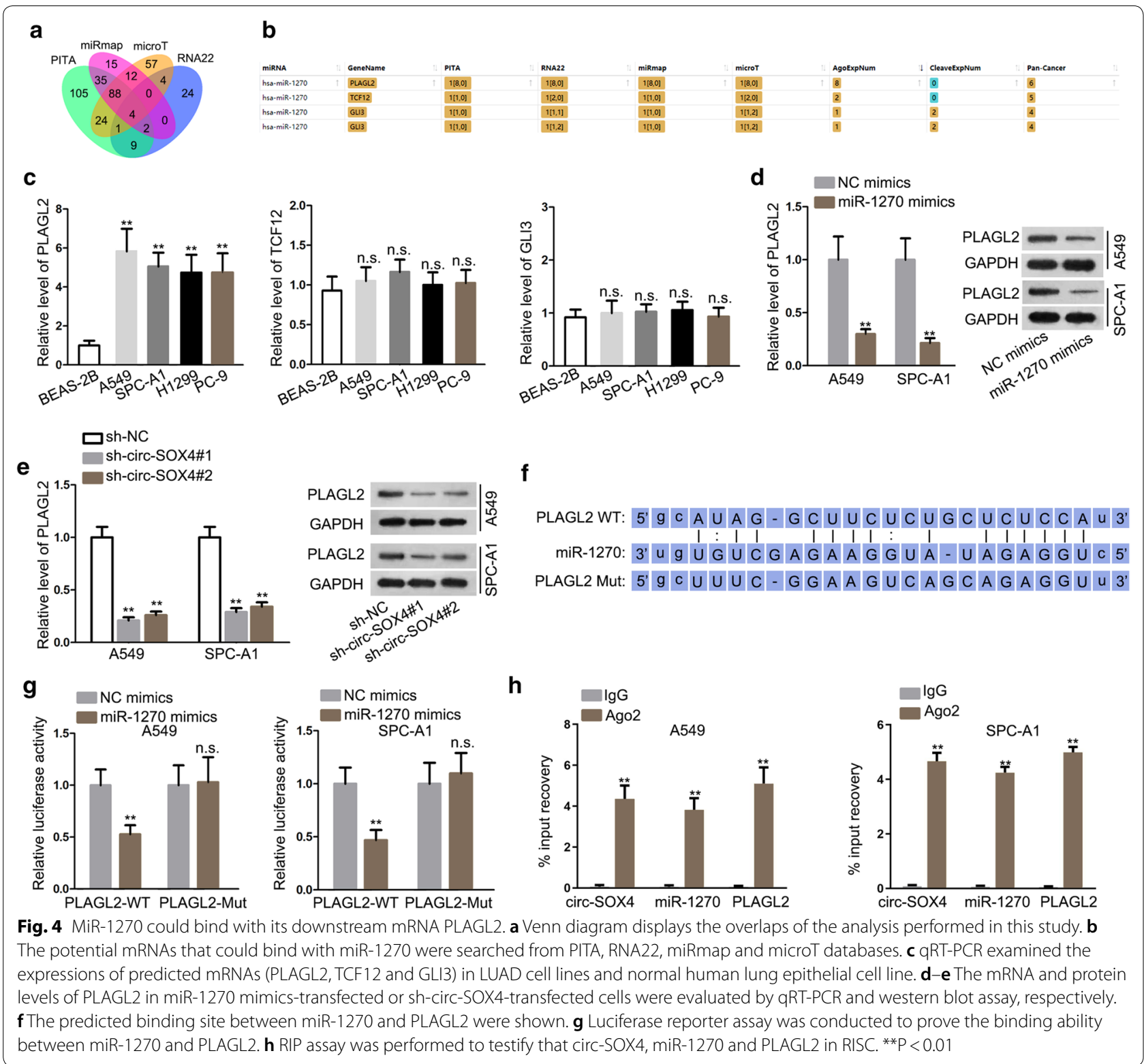

Mechanistically, circRNAs have been reported as sponges for miRNAs, thus functioning as promoter or inhibitor in carcinogenesis. In our current study, we observed that circ-SOX4 was predominantly located in the cytoplasm of LUAD cells. Based on bioinformatics analysis, miRNAs that were potentially interacted with circ-SOX4 were predicted. Among which, miR-1270 had highest enrichment in LUAD cells. Thus, it was selected as the next research object. MiR-1270 was reported in a previous study as its tumor-suppressive role [42]. In this research, it was found that circ-SOX4 could directly bind to miR-1270 and miR-1270 expression was negatively regulated by circ-SOX4 in LUAD. Briefly, circ-SOX4 facilitated LUAD development by serving as a sponge for miR-1270.

Previous studies have certified that circRNAs affect the development of cancer by sponging miRNAs to regulate mRNAs [43, 44]. For illustration, circGFRA1 sponges miR-34a and promotes the progression of breast cancer by modulating GFRA1 [45]. Hsa-circ-0045714 regulates chondrocyte viability and apoptosis by enhancing miR-193b expression and targeting IGF1R [46]. According to mechanism investigation, we confirmed that miR-1270 could interact with PLAGL2. PLAGL2 has been confirmed as an oncogene in colorectal cancer [47] and bladder urothelial 


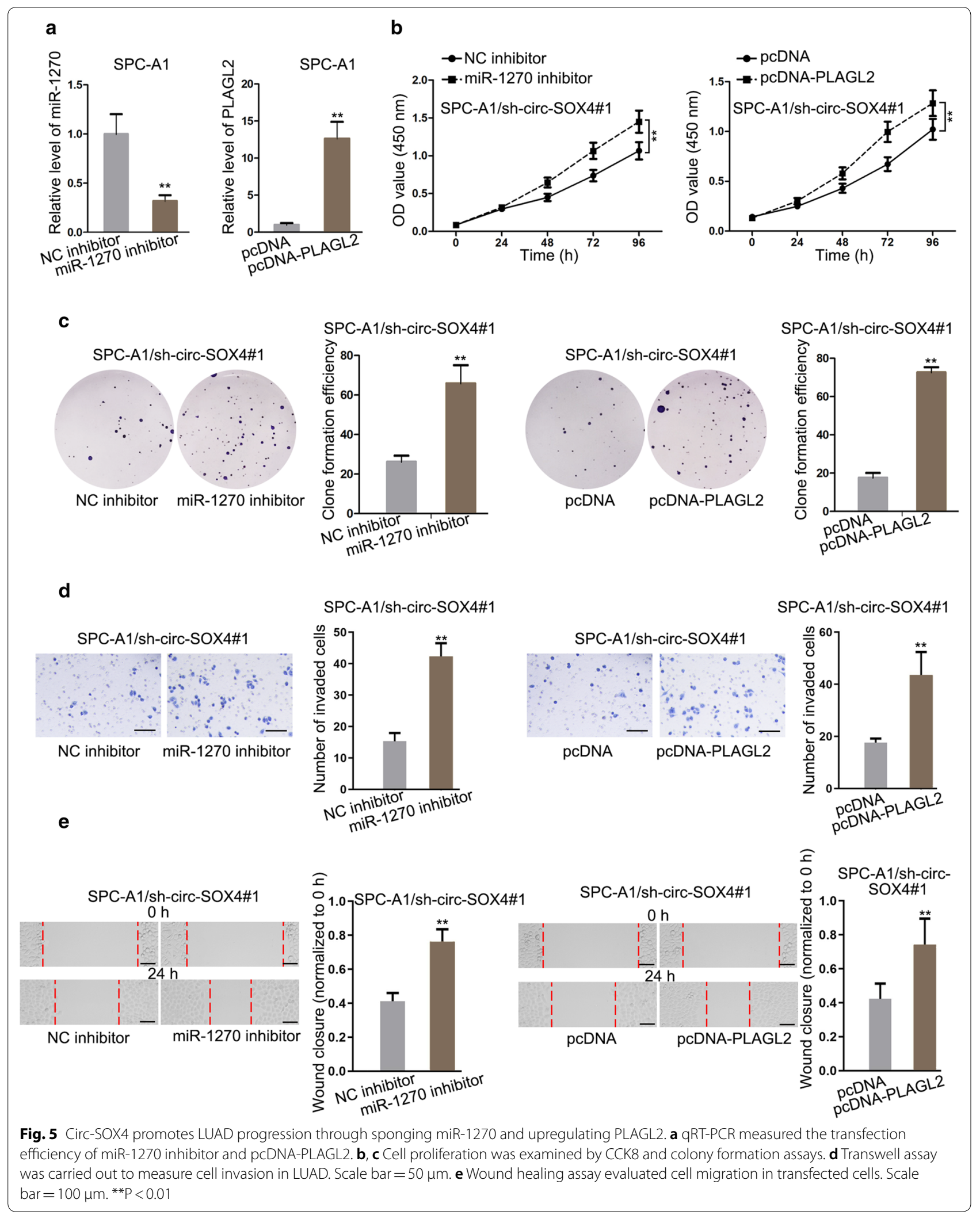




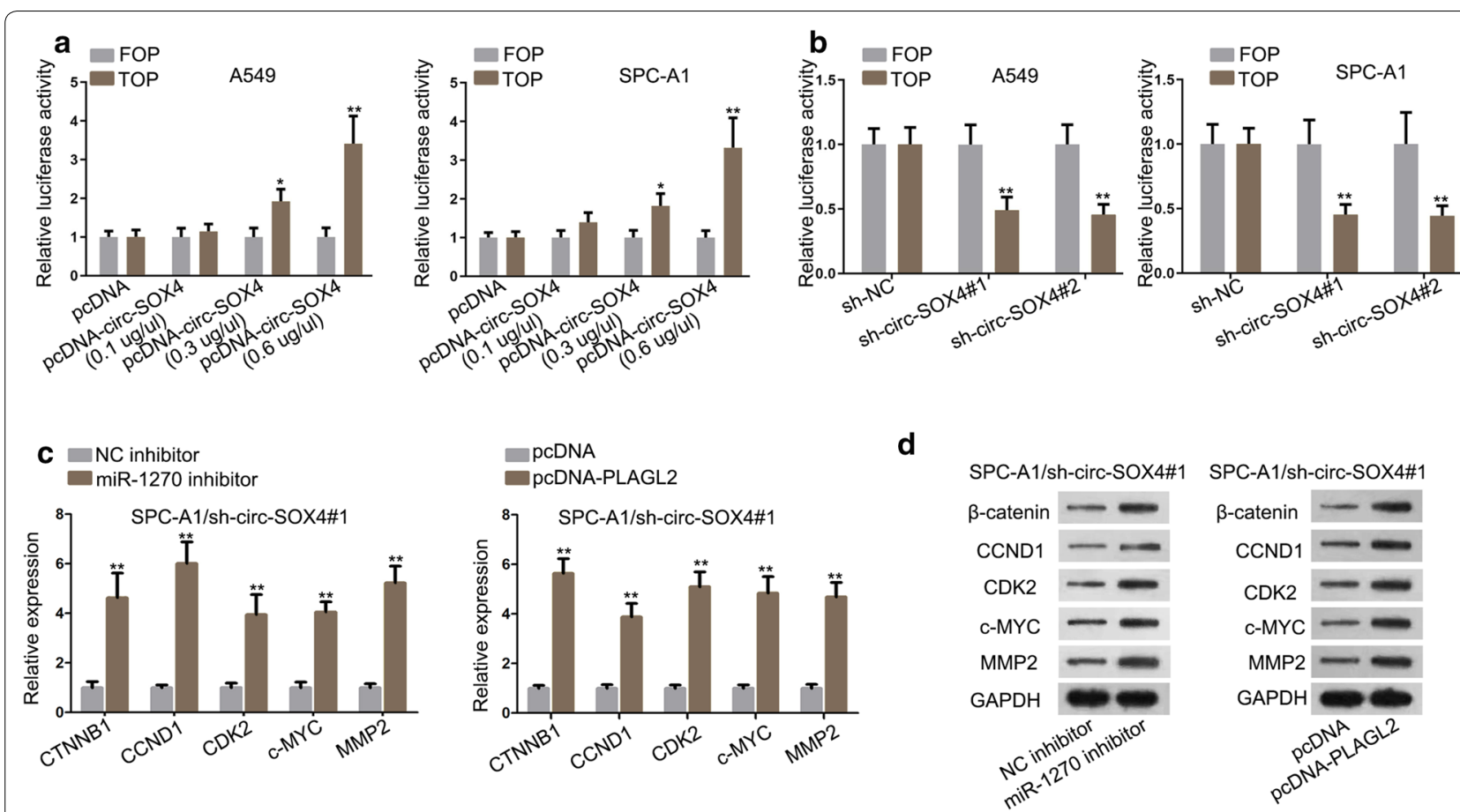

Fig. 6 Circ-SOX4 promotes LUAD progression through miR-1270/PLAGL2/WNT/ $\beta$-catenin signaling pathway. a TOP/FOP flash assay evaluated the influences of different concentration of pcDNA3.1/PLAGL2 on the luciferase activity of TOP and FOP reporters. $\mathbf{b}$ The luciferase activities of TOP and FOP reporters in sh-NC and sh-circ-SOX4 groups were assessed by TOP/FOP flash assay. $\mathbf{c}, \mathbf{d}$ qRT-PCR and western blot assay, respectively evaluated the level of WNT pathway-related mRNAs and proteins in SPC-A1/sh-circ-SOX4 cells. ${ }^{*} \mathrm{P}<0.05,{ }^{* *} \mathrm{P}<0.01$

carcinoma [48]. According to the results in this study, PLAGL2 expression was negatively regulated by miR1270 and positively regulated by circ-SOX4 in LUAD cells. Importantly, PLAGL2 was reported to activate WNT pathway [49]. Thus, we further investigated that circ-SOX4 could activate Wnt pathway via upregulation of PLAGL2. Through rescue assays, we found that miR-1270 repression or PLAGL2 elevation reversed the inhibitory role of circ-SOX4 silencing in LUAD cell growth and Wnt pathway.

\section{Conclusion}

In conclusion, our study revealed that circ-SOX4 boosted LUAD progression by targeting miR-1270/PLAGL2 axis and activating Wnt pathway. Above results in present study might provide the meaningful revelation for investigating the novel therapeutic methods for LUAD patients.

\section{Supplementary information}

Supplementary information accompanies this paper at https://doi. org/10.1186/s12935-019-1065-x.
Additional file 1: Figure S1. (A) SOX4 expression was tested in sh-circSOX4 transfected cells. (B) Western blot assay was performed to assess the protein levels of CD44, E-cadherin and N-cadherin. n.s.: no significance. ${ }^{* *} \mathrm{P}<0.01$

\section{Abbreviations}

LUAD: lung adenocarcinoma; circRNA: circular RNA; NSCLC: non-small cell lung cancer; SOX4: SRY-box transcription factor 4; PLAGL2: PLAG1 like zinc finger 2; shRNA: short hairpin RNA; FBS: fetal bovine serum; NC: negative control; GAPDH: glyceraldehyde-3-phosphate dehydrogenase; CCK-8: cell counting kit-8; PBS: phosphate buffer saline; qRT-PCR: quantitative real-time polymerase chain reaction; IgG: immunoglobulin G; PVDF: polyvinylidene fluoride; SD: standard deviation; RISC: RNA-induced silencing complex; miRNAs: microRNAs,

\section{Acknowledgements}

We appreciate the supports of China-Japan Union Hospital of Jilin University.

\section{Authors' contributions}

NG devoted to research design and data curations. NG and BY were responsible for experiment and figures. All authors read and approved the final manuscript.

\section{Funding}

None.

Availability of data and materials

Research data and material are not shared.

Ethics approval and consent to participate

The procedures of the human and animals experiments were authorized by China-Japan Union Hospital of Jilin University. And all involved participants signed informed consent. 


\section{Consent for publication}

All authors have approved the publication of this manuscript.

\section{Competing interests}

The authors declare that they have no competing interests.

\section{Author details}

${ }^{1}$ Department of Thoracic Surgery, China-Japan Union Hospital of Jilin University, Changchun 130033, Jilin, China. ${ }^{2}$ Department of Anesthesiology, China-Japan Union Hospital of Jilin University, No. 126 XianTai Road, Changchun 130033, Jilin, China.

Received: 17 July 2019 Accepted: 10 December 2019

Published online: 03 January 2020

\section{References}

1. Smith RA, Manassaram-Baptiste D, Brooks D, Doroshenk M, Fedewa S, Saslow D, Brawley OW, Wender R. Cancer screening in the United States, 2015: a review of current American cancer society guidelines and current issues in cancer screening. CA Cancer J Clin. 2015;65(1):30-54.

2. Ettinger DS, Wood DE, Akerley W, Bazhenova LA, Borghaei H, Camidge, Cheney RT, Chirieac LR, D'Amico TA, Demmy TL, et al. Non-small cell lung cancer, version 6. J Natl Compr Cancer Netw. 2015;13(5):515-24.

3. Lv S, Xue J, Wu C, Wang L, Wu J, Xu S, Liang X, Lou J. Identification of a panel of serum microRNAs as biomarkers for early detection of lung adenocarcinoma. J Cancer. 2017:8(1):48-56.

4. Riaz SP, Luchtenborg M, Coupland VH, Spicer J, Peake MD, Moller H. Trends in incidence of small cell lung cancer and all lung cancer. Lung Cancer. 2012;75(3):280-4.

5. Deffebach ME, Humphrey L. Lung cancer screening. Surg Clin N Am. 2015:95(5):967-78.

6. Li M, Qiu M, Xu Y, Mao Q, Wang J, Dong G, Xia W, Yin R, Xu L. Differentially expressed protein-coding genes and long noncoding RNA in early-stage lung cancer. Tumour Biol. 2015;36(12):9969-78.

7. He J, Xie Q, Xu H, Li J, Li Y. Circular RNAs and cancer. Cancer Lett. 2017;396:138-44.

8. Meng S, Zhou H, Feng Z, Xu Z, Tang Y, Li P, Wu M. CircRNA: functions and properties of a novel potential biomarker for cancer. Mol Cancer. 2017;16(1):94

9. Jin X, Feng CY, Xiang Z, Chen YP, Li YM. CircRNA expression pattern and circRNA-miRNA-mRNA network in the pathogenesis of nonalcoholic steatohepatitis. Oncotarget. 2016;7(41):66455-67.

10. Qu S, Yang X, Li X, Wang J, Gao Y, Shang R, Sun W, Dou K, Li H. Circular RNA: a new star of noncoding RNAs. Cancer Lett. 2015;365(2):141-8.

11. Cui X, Niu W, Kong L, He M, Jiang K, Chen S, Zhong A, Li W, Lu J, Zhang L. hsa_circRNA_103636: potential novel diagnostic and therapeutic biomarker in Major depressive disorder. Biomark Med. 2016;10(9):943-52.

12. Mao W, Huang X, Wang L, Zhang Z, Liu M, Li Y, Luo M, Yao X, Fan J, Geng J. Circular RNA hsa_circ_0068871 regulates FGFR3 expression and activates STAT3 by targeting miR-181a-5p to promote bladder cancer progression. J Exp Clin Cancer Res. 2019;38(1):169.

13. Xu L, Feng X, Hao X, Wang P, Zhang Y, Zheng X, Li L, Ren S, Zhang M, Xu M. CircSETD3 (Hsa_circ_0000567) acts as a sponge for microRNA-421 inhibiting hepatocellular carcinoma growth. J Exp Clin Cancer Res. 2019;38(1):98

14. Yang F, Liu DY, Guo JT, Ge N, Zhu P, Liu X, Wang S, Wang GX, Sun SY. Circular RNA circ-LDLRAD3 as a biomarker in diagnosis of pancreatic cancer. World J Gastroenterol. 2017;23(47):8345-54.

15. Yao Y, Hua Q, Zhou Y, Shen H. CircRNA has_circ_0001946 promotes cell growth in lung adenocarcinoma by regulating miR-135a-5p/SIRT1 axis and activating Wnt/beta-catenin signaling pathway. Biomed Pharmacother. 2019;111:1367-75.

16. Yao Y, Hua Q, Zhou Y. CircRNA has_circ_0006427 suppresses the progression of lung adenocarcinoma by regulating miR-6783-3p/DKK1 axis and inactivating Wnt/beta-catenin signaling pathway. Biochem Biophys Res Commun. 2019;508(1):37-45.

17. Igaz P, Igaz I, Nagy Z, Nyiro G, Szabo PM, Falus A, Patocs A, Racz K. MicroRNAs in adrenal tumors: relevance for pathogenesis, diagnosis, and therapy. Cell Mol Life Sci. 2015;72(3):417-28.
18. Chen L, Gao H, Liang J, Qiao J, Duan J, Shi H, Zhen T, Li H, Zhang F, Zhu Z, et al. miR-203a-3p promotes colorectal cancer proliferation and migration by targeting PDE4D. Am J Cancer Res. 2018;8(12):2387-401.

19. Deng Y, Li Y, Fang Q, Luo H, Zhu G. microRNA-744 is downregulated in glioblastoma and inhibits the aggressive behaviors by directly targeting NOB1. Am J Cancer Res. 2018;8(11):2238-53.

20. Zhang Y, Liu H, Li W, Yu J, Li J, Shen Z, Ye G, Qi X, Li G. CircRNA_100269 is downregulated in gastric cancer and suppresses tumor cell growth by targeting miR-630. Aging. 2017;9(6):1585-94

21. Liu B, Ye B, Yang L, Zhu X, Huang G, Zhu P, Du Y, Wu J, Qin X, Chen R, et al. Long noncoding RNA IncKdm $2 b$ is required for ILC3 maintenance by initiation of Zfp292 expression. Nat Immunol. 2017;18(5):499-508.

22. Wu Y, Zhang Y, Zhang Y, Wang JJ. CircRNA hsa_circ_0005105 upregulates NAMPT expression and promotes chondrocyte extracellular matrix degradation by sponging miR-26a. Cell Biol Int. 2017;41(12):1283-9.

23. Zhang XL, Xu LL, Wang F. Hsa_circ_0020397 regulates colorectal cancer cell viability, apoptosis and invasion by promoting the expression of the miR-138 targets TERT and PD-L1. Cell Biol Int. 2017;41(9):1056-64.

24. YiT, Zhou X, Sang K, Zhou J, Ge L. MicroRNA-1270 modulates papillary thyroid cancer cell development by regulating SCAI. Biomed Pharmacother. 2019;109:2357-64.

25. Zhong L, Zheng C, Fang H, Xu M, Chen B, Li C. MicroRNA-1270 is associated with poor prognosis and its inhibition yielded anticancer mechanisms in human osteosarcoma. IUBMB Life. 2018;70(7):625-32.

26. Huang W, Lu Y, Wang F, Huang X, Yu Z. Downregulation of circular RNA hsa_circ_0000144 inhibits bladder cancer progression via stimulating miR-217 and suppressing RUNX2 expression. Gene. 2018;678:337-42.

27. Yong W, Zhuoqi X, Baocheng W, Dongsheng Z, Chuan Z, Yueming S. Hsa_circ_0071589 promotes carcinogenesis via the miR-600/EZH2 axis in colorectal cancer. Biomed Pharmacother. 2018;102:1188-94.

28. Wang YP, Guo PT, Zhu Z, Zhang H, Xu Y, Chen YZ, Liu F, Ma SP. Pleomorphic adenoma gene like-2 induces epithelial-mesenchymal transition via Wnt/beta-catenin signaling pathway in human colorectal adenocarcinoma. Oncol Rep. 2017;37(4):1961-70.

29. Funato Y, Miki H. Redox regulation of Wnt signalling via nucleoredoxin. Free Radic Res. 2010:44(4):379-88.

30. Yoshida GJ, Saya H. Inversed relationship between CD44 variant and c-Myc due to oxidative stress-induced canonical Wnt activation. Biochem Biophys Res Commun. 2014;443(2):622-7.

31. Karimaian A, Majidinia M, Bannazadeh Baghi H, Yousefi B. The crosstalk between Wnt/beta-catenin signaling pathway with DNA damage response and oxidative stress: implications in cancer therapy. DNA Repair. 2017;51:14-9.

32. Yoshida GJ. The heterogeneity of cancer stem-like cells at the invasive front. Cancer Cell Int. 2017;17:23.

33. Aggarwal A, Lewison G, Idir S, Peters M, Aldige C, Boerckel W, Boyle P, Trimble EL, Roe P, Sethi T, et al. The state of lung cancer research: a global analysis. JThorac Oncol. 2016;11(7):1040-50.

34. Xu X, Zhu Y, Liang Z, Li S, Xu X, Wang X, Wu J, Hu Z, Meng S, Liu B, et al. $c-$ Met and CREB1 are involved in miR-433-mediated inhibition of the epithelial-mesenchymal transition in bladder cancer by regulating Akt/ GSK-3beta/Snail signaling. Cell Death Dis. 2016;7:e2088.

35. Wang $X$, Zhang $Y$, Huang L, Zhang J, Pan F, Li B, Yan Y, Jia B, Liu H, Li S, et al. Decreased expression of hsa_circ_001988 in colorectal cancer and its clinical significances. Int J Clin Exp Pathol. 2015;8(12):16020-5.

36. Xie H, Ren X, Xin S, Lan X, Lu G, Lin Y, Yang S, Zeng Z, Liao W, Ding YQ, et al. Emerging roles of circRNA_001569 targeting miR-145 in the proliferation and invasion of colorectal cancer. Oncotarget. 2016;7(18):26680-91.

37. Zhong Z, LV M, Chen J. Screening differential circular RNA expression profiles reveals the regulatory role of circTCF25-miR-103a-3p/miR-107-CDK6 pathway in bladder carcinoma. Sci Rep. 2016;6:30919.

38. Xu L, Zhang M, Zheng X, Yi P, Lan C, Xu M. The circular RNA ciRS-7 (Cdr1as) acts as a risk factor of hepatic microvascular invasion in hepatocellular carcinoma. J Cancer Res Clin Oncol. 2017;143(1):17-27.

39. Tang Q, Chen Z, Zhao L. Circular RNA hsa_circ_0000515 acts as a miR-326 sponge to promote cervical cancer progression through up-regulation of ELK1. Aging. 2019;11(22):9982-99.

40. Zhou J, Zhang S, Chen Z, He Z, Xu Y, Li Z. CircRNA-ENO1 promoted glycolysis and tumor progression in lung adenocarcinoma through upregulating its host gene ENO1. Cell Death Dis. 2019;10(12):885. 
41. Huang X, He M, Huang S, Lin R, Zhan M, Yang D, Shen H, Xu S, Cheng W, Yu J, et al. Circular RNA circERBB2 promotes gallbladder cancer progression by regulating PA2G4-dependent rDNA transcription. Mol Cancer. 2019;18(1):166.

42. Wei L, Li P, Zhao C, Wang N, Wei N. Upregulation of microRNA-1270 suppressed human glioblastoma cancer cell proliferation migration and tumorigenesis by acting through WT1. OncoTargets Ther. 2019;12:4839-48.

43. Xu XW, Zheng BA, Hu ZM, Qian ZY, Huang CJ, Liu XQ, Wu WD. Circular RNA hsa_circ_000984 promotes colon cancer growth and metastasis by sponging miR-106b. Oncotarget. 2017;8(53):91674-83.

44. Zhong Z, Huang M, Lv M, He Y, Duan C, Zhang L, Chen J. Circular RNA MYLK as a competing endogenous RNA promotes bladder cancer progression through modulating VEGFANEGFR2 signaling pathway. Cancer Lett. 2017:403:305-17.

45. Wang Y, Chen F, Zhao M, Yang Z, Li J, Zhang S, Zhang W, Ye L, Zhang X. The long noncoding RNA HULC promotes liver cancer by increasing the expression of the HMGA2 oncogene via sequestration of the microRNA-186. J Biol Chem. 2017;292(37):15395-407.
46. Li BF, Zhang Y, Xiao J, Wang F, Li M, Guo XZ, Xie HB, Xia H, Chen B. Hsa_circ_0045714 regulates chondrocyte proliferation, apoptosis and extracellular matrix synthesis by promoting the expression of miR-193b target gene IGF1R. Hum Cell. 2017;30(4):311-8.

47. Li N, Li D, Du Y, Su C, Yang C, Lin C, Li X, Hu G. Overexpressed PLAGL2 transcriptionally activates Wnt6 and promotes cancer development in colorectal cancer. Oncol Rep. 2019;41(2):875-84.

48. Qu G, Xu Y, Wan SP, Yang G. Expression of PLAGL2 in bladder urothelial carcinoma and its relationship to lymph node metastasis and survival. Sci Rep. 2018;8(1):6044.

49. Zhou J, Liu H, Zhang L, Liu X, Zhang C, Wang Y, He Q, Zhang Y, Li Y, Chen $\mathrm{Q}$, et al. DJ-1 promotes colorectal cancer progression through activating PLAGL2/Wnt/BMP4 axis. Cell Death Dis. 2018;9(9):865.

\section{Publisher's Note}

Springer Nature remains neutral with regard to jurisdictional claims in published maps and institutional affiliations.
Ready to submit your research? Choose BMC and benefit from:

- fast, convenient online submission

- thorough peer review by experienced researchers in your field

- rapid publication on acceptance

- support for research data, including large and complex data types

- gold Open Access which fosters wider collaboration and increased citations

- maximum visibility for your research: over $100 \mathrm{M}$ website views per year

At BMC, research is always in progress.

Learn more biomedcentral.com/submissions 